\title{
The initial-boundary value problem for a class of third order pseudoparabolic equations
}

\author{
Yanqing Feng ${ }^{1 *} \mathbb{D}$, Limin Guo ${ }^{1}$ and Zhongying Wang ${ }^{1}$
}

"Correspondence: fengyq@czu.cn 'School of Science, Changzhou Institute of Technology, Changzhou, People's Republic of China

\begin{abstract}
In this paper, a priori estimate for a linear third pseudoparabolic operator with bound is established, and applying the above result, the existence and uniqueness theorem of solutions for a class of nonlinear pseudoparabolic equations is obtained with the help of the homeomorphism method and the initial value method. Furthermore, an existence and uniqueness theorem of the semilinear equation is obtained as a corollary.
\end{abstract}

Keywords: Third order pseudoparabolic equations; Homeomorphism method; Initial-boundary value problem; Existence and uniqueness theorem

\section{Introduction}

Consider the initial-boundary value problem

$$
\begin{aligned}
& \left(a_{i, j}\left(u+u_{t}\right)_{x_{i}}\right)_{x_{j}}+b_{i} u_{x_{i}}-u_{t}+F\left(x, t, u, u_{x_{i}}\right)=f(x, t, u), \quad(x, t) \in D, \\
& \left.u\right|_{t=0}=0, \quad x \in \Omega, \\
& \left.u\right|_{\partial \Omega}=0, \quad(x, t) \in \partial \Omega \times[0, T],
\end{aligned}
$$

where $\Omega$ is a connected bounded subset of $n$-dimensional space, the boundary of $\Omega$ is piecewise smooth and has nonnegative mean curvature everywhere, $D=\Omega \times[0, T]$ belongs to the Hilbert space $W_{2}^{2,1}(D)$, and $a_{i, j}, b_{i}$ are bounded measurable functions.

Using a continuous method, Sigillito [1] explored the solution for the heat equation. Elcart and Sigillito [2,3] had derived an explicit coercivity inequality and discussed the convergence of the algorithm for a semilinear third order pseudoparabolic equation of the following type:

$$
\Delta\left(u+u_{t}\right)-u_{t}=f(x, t, u), \quad(x, t) \in D,
$$

since then, there have been some further studies of other forms of parabolic equations, most of the results have focused on the discussion of algorithms [4].

(c) The Author(s) 2020. This article is licensed under a Creative Commons Attribution 4.0 International License, which permits use, sharing, adaptation, distribution and reproduction in any medium or format, as long as you give appropriate credit to the original author(s) and the source, provide a link to the Creative Commons licence, and indicate if changes were made. The images or other third party material in this article are included in the article's Creative Commons licence, unless indicated otherwise in a credit line to the material. If material is not included in the article's Creative Commons licence and your intended use is not permitted by statutory regulation or exceeds the permitted use, you will need to obtain permission directly from the copyright holder. To view a copy of this licence, visit http://creativecommons.org/licenses/by/4.0/. 
In 2004, Bouziani $[5,6]$ had derived an explicit coercivity inequality and given a sufficient condition for the existence and uniqueness of a solution to the first order parabolic equation.

Motivated by the spirit of this work and results by Brown and Lin [7], the explicit coercivity inequalities of a linear third pseudoparabolic operator with bound are obtained in Sect. 3. By using these estimates, we shall utilize the homeomorphism method and the initial value method to give a new set of sufficient conditions for the existence and uniqueness of the third order pseudoparabolic equation in this paper, which can be found in Sect. 4.

\section{Preliminaries and lemmas}

In this section, we will state some lemmas which are useful to our results.

Firstly, we will give sufficient conditions for $f$ to be a global homeomorphism of $D$ onto $Y$.

Definition 2.1 ([8]) Let $X, Y$ be Banach spaces, $D \subseteq X$ be open and connected, the continuous mapping $f: D \subset X \rightarrow Y$ satisfies condition (C) if and only if for any continuous function $r:[0, a) \rightarrow D \subseteq X$ such that

$$
f(r(t))=q(t), \quad t \in[0, a)
$$

where $q:[0,1] \rightarrow Y$ is any line in $Y$, there is a sequence $\left\{t_{n}\right\}$ such that $t_{n} \rightarrow a, n \rightarrow \infty$ and

$$
\lim _{n \rightarrow \infty} r\left(t_{n}\right)
$$

exists and is in $D$.

In the following for convenience, with no loss of generality, for the function $q$ one may assume that $q(t)=(1-t) f\left(x_{0}\right)+t y, t \in[0,1]$, for arbitrary $x_{0} \in D$ and $y \in Y$.

Theorem 2.1 (Plasctock [8]) Let $f: D \subset X \rightarrow Y$ be a local homeomorphism.

Then $f$ is a global homeomorphism of D onto $Y$ if and only iff satisfies condition (C).

Secondly, the comparison theorem plays an important role to prove the sufficient condition for the existence of a unique solution of the problem (1).

Let $E$ be an open $(t, x)$-set in $R^{2}$ and $g \in C[E, R]$. Consider the scalar differential equation with an initial condition

$$
\left\{\begin{array}{l}
u^{\prime}=g(t, u), \\
u\left(t_{0}\right)=u_{0} .
\end{array}\right.
$$

Definition 2.2 ([9]) Let $y(t)$ be a solution of the scalar differential equation (4) on $\left[t_{0}, t_{0}+a\right)$, then $y(t)$ is said to be a maximal solution of (4) if, for every solution $u(t)$ of (4) existing on $\left[t_{0}, t_{0}+a\right)$, we have the inequality

$$
u(t) \leq y(t), \quad t \in\left[t_{0}, t_{0}+a\right)
$$

holds. 
Theorem 2.2 (Plasctock [9]) Let $g \in C\left[R_{0}, R\right]$, where $R_{0}$ is the rectangle $t_{0} \leq t \leq t_{0}+a$, $\left|u-u_{0}\right| \leq b$, and $|g(t, u)| \leq M$ on $R_{0}$.Then there exist a maximal solution and a minimal solution of (4) on $\left[t_{0}, t_{0}+a\right]$, where $\alpha=\min (a, b \backslash 2(M+b))$.

Theorem 2.3 (Comparison theorem [9]) In the setting of the above, suppose that $\left[t_{0}, t_{0}+b\right)$ is the largest interval in which the maximal solution $y(t)$ of (4) exists. Let

$$
m \in C\left[\left[t_{0}, t_{0}+b\right), R\right], \quad(t, m(t)) \in E \quad \text { for } t \in\left[t_{0}, t_{0}+b\right), m\left(t_{0}\right) \leq u_{0},
$$

and for a fixed Dini derivative

$$
D m(t) \leq g(t, m(t)), \quad t \in\left[t_{0}, t_{0}+b\right) \backslash T,
$$

then

$$
m(t) \leq y(t), \quad t \in\left[t_{0}, t_{0}+b\right)
$$

where $T$ denotes an almost countable subset of $t \in\left[t_{0}, t_{0}+b\right)$.

\section{The coercivity inequality}

Let $W_{0}(D)$ denote the Hilbert space with the norm

$$
\left(\|u\|_{2,1}\right)^{2}=\int_{D}\left(u^{2}+|\nabla u|^{2}+\left|D^{2} u\right|^{2}+u_{t}^{2}\right) d x d t
$$

here $\left|D^{2} u\right|^{2}$ represents the sum of the squares of all the second derivatives with respect to space variables. In this section we derive a coercivity inequality,

$$
|\|u\|| \leq \text { const }\|L u\|
$$

for the pseudoparabolic operator defined by

$$
L u=\left(a_{i j}\left(u+u_{t}\right)_{x_{i}}\right)_{x_{j}}+b_{i} u_{x_{i}}-a u-u_{t} .
$$

The norm $|\|\cdot\||$ on $W_{0}$ is defined by

$$
\left[\left(\|u\|_{2,1}\right)^{2}+\int_{0}^{T}\left\|u_{t}\right\|_{2}^{2} d t\right]^{\frac{1}{2}}
$$

where $\|\cdot\|_{2}$ is the norm on $W_{2}^{2}(\Omega),\|\cdot\|$ is the norm on $L_{2}(D), a: W_{0}(D) \rightarrow L_{2}(D)$ is continuous and a bounded function on $t, x_{1}, \ldots, x_{n}, u$.

We assume that $a_{i j}$ is a symmetric matrix of measurable functions satisfying the inequality

$$
\tau^{2}|\xi| \leq a_{i, j} \xi_{i} \xi_{j}
$$


for some positive constant $\tau$, all $n$-dimensional vectors $\xi$ and all $x$ in $D$. We also assume that the functions $a_{i j}$ are sufficiently regular to ensure the validity of the identity

$$
\int_{\Omega} u\left(a_{i j} u_{x_{j}}\right)_{x_{i}} d x=-\int_{\Omega} a_{i j} u_{x_{j}} u_{x_{i}} d x
$$

for $u$ in $W_{2}^{2}(\Omega)$. From (5), we have

$$
\begin{aligned}
-u L u & =-u\left(a_{i, j}\left(u+u_{t}\right)_{x_{i}}\right)_{x_{j}}-b_{i} u_{x_{i}} u+a u^{2}+u_{t} u \\
& =\left(a_{i, j}\right)_{x_{j}}\left(u+u_{t}\right)_{x_{i}} u+a_{i, j}\left(u+u_{t}\right)_{x_{i}}\left(u+u_{t}\right)_{x_{j}}-b_{i} u_{x_{i}} u+a u^{2}+u_{t} u .
\end{aligned}
$$

Using the inequality

$$
2 b c \leq \varepsilon b^{2}+\varepsilon^{-1} c^{2}
$$

and the inequality

$$
\tau^{2}|\xi| \leq a_{i, j} \xi_{i} \xi_{j}
$$

for $\varepsilon>0, \alpha>0$, the inequality

$$
\begin{aligned}
\int_{D}(L u)^{2} d x d t \geq & 2 \varepsilon \tau^{2}(1-\alpha) \int_{D}|\nabla u|^{2} d x d t d u \\
& +\left(a_{0}-\frac{\varepsilon}{2}-\frac{S^{2}}{4 \tau^{2} \alpha}\right) 2 \varepsilon \int_{D} u^{2} d x d t
\end{aligned}
$$

is valid for $u$ in $W_{0}$.

The next two lemmas are obtained from (5) by evident choices of $\varepsilon$ and $\alpha$. In order to facilitate statements to be made below, we define $S=\sup \left|b_{i}-\left(a_{i, j}\right)_{x_{j}}\right|, a_{0}=\inf _{D} a(x, t)$.

Lemma 3.1 The inequality

$$
\int_{D}(L u)^{2} d x d t \geq\left(a_{0}-\frac{S^{2}}{4 \tau^{2}}\right)^{2} \int_{D} u^{2} d x d t
$$

is valid for $u$ in $W_{0}$.

Lemma 3.2 The inequality

$$
\int_{D}(L u)^{2} d x d t \geq\left(2 \tau \sqrt{a_{0}(1-S)}\right)^{2} \int_{D}|\nabla u|^{2} d x d t
$$

is valid for $u$ in $W_{0}$.

We define

$$
P u=a_{i, j} u_{x_{j}} u_{x_{i}}, \quad Q u=\left(a_{i, j} u_{x_{i}}\right)_{x_{j}}, \quad a_{1}=\sup \left|b_{i}\right|, \quad \bar{M}=\max _{i} \sum_{j} a_{i, j}^{2},
$$


and from [2] we have the inequality

$$
\frac{\tau^{2}}{2} \int_{D} \sum\left|D^{2} u\right|^{2} d x d t \leq \int_{D} P^{2} u d x d t+\frac{n^{4} B^{2}}{2 \tau^{2}} \int_{D}|\nabla u|^{2} d x d t
$$

From $P u=Q u-\left(a_{i, j}\right)_{x_{j}} u_{x_{i}}, u \in W_{0}$, we have

$$
\begin{aligned}
\int_{D} P^{2} u d x d t & \leq 2 \int_{D} Q^{2} u d x d t+2 \int_{D}\left(\left(a_{i, j}\right)_{x_{j}} u_{x_{i}}\right)^{2} d x d t \\
& \leq 2 \int_{D} Q^{2} u d x d t+2 \bar{M} \int_{D}|\nabla u|^{2} d x d t
\end{aligned}
$$

so

$$
\begin{aligned}
\frac{\tau^{2}}{2} \int_{D}\left|D^{2} u\right|^{2} d x d t \leq & 2 \int_{D} Q^{2} u d x d t+\left(\frac{n^{4} B^{2}}{2 \tau^{2}}+2 \bar{M}\right) \int_{D}|\nabla u|^{2} d x d t \\
\leq & 2 \int_{D} Q^{2}\left(u+u_{t}\right) d x d t \\
& +\left(\frac{n^{4} B^{2}}{2 \tau^{2}}+2 \bar{M}\right) \int_{D}|\nabla u|^{2} d x d t .
\end{aligned}
$$

Remark 3.1 Results analogous in the present situation are in Lemma 3.3.

Lemma 3.3 The inequality

$$
\begin{aligned}
\frac{\tau^{2}}{2} \int_{D}\left|D^{2} u\right|^{2} d x d t \leq & 2 \int_{D} Q^{2}\left(u+u_{t}\right) d x d t \\
& +\left(\frac{n^{4} B^{2}}{2 \tau^{2}}+2 \bar{M}\right) \int_{D}|\nabla u|^{2} d x d t
\end{aligned}
$$

is valid for $u$ in $W_{0}$.

From $Q u=P u+\left(a_{i, j}\right)_{x_{j}} u_{x_{i}}$, we also have

$$
\begin{aligned}
\int_{D} Q^{2}\left(u+u_{t}\right) d x d t \leq & \int_{D}(L u)^{2} d x d t+\left(\frac{1}{4}+\frac{S}{2}+a_{1}\right) \int_{D} u^{2} d x d t \\
& +\frac{S}{2} \int_{D}|\nabla u|^{2} d x d t
\end{aligned}
$$

The inequalities (10) and (11) imply that

$$
\begin{aligned}
\frac{\tau^{2}}{2} \int_{D}\left|D^{2} u\right|^{2} d x d t \leq & \int_{D}(L u)^{2} d x d t+\left(\frac{1}{4}+\frac{S}{2}+a_{1}\right) \\
& \int_{D} u^{2} d x d t+\left(\frac{S}{2}+\frac{n^{4} B^{2}}{2 \tau^{2}}+2 \bar{M}\right) \int_{D}|\nabla u|^{2} d x d t
\end{aligned}
$$

Denote

$$
L_{0} u=\left(a_{i, j}\left(u+u_{t}\right)_{x_{i}}\right)_{x_{j}}+b_{i} u_{x_{i}}-a u,
$$


and by further application of the arithmetic-geometric mean inequality to

$$
-\int_{D} u u_{t} d x d t=-\int_{D} u L u d x d t+\int_{D} u L_{0} u d x d t
$$

we obtain

$$
\int_{D} u_{t}^{2} d x d t \leq \int_{D}(L u)^{2} d x d t-4\left(a_{0}+\frac{1}{2}+\frac{S}{4 \tau^{2}}\right) \int_{D} u^{2} d x d t
$$

Combining (8), (9), (12) and (13), we have Lemma 3.4.

Lemma 3.4 If $a_{0}-\frac{S^{2}}{4 \tau^{2}}>0$, the inequality

$$
|\|u\|| \leq C\|L u\|
$$

is valid for $u$ in $W_{0}$, where

$$
C^{2}=\frac{2+\tau^{2}}{\tau^{2}}+\frac{\tau^{2}+S+4 \bar{M}}{4 \tau^{4} a_{0}(1-S)}+\frac{B^{2} n^{4}}{4 \tau^{6} a_{0}(1-S)}+\frac{8 \tau^{2}\left(1+4 a_{1}\right)-16 \tau^{4}\left(4 a_{0}+1\right)}{\left(4 a_{0} \tau^{2}-S^{2}\right)^{2}} .
$$

\section{The coercivity inequality}

Denote

$$
M u=\left(a_{i, j}\left(u+u_{t}\right)_{x_{i}}\right)_{x_{j}}+b_{i} u_{x_{i}}-u_{t},
$$

then $M$ is a linear operator from $W_{0}(D)$ to $L_{2}(D)$. Now let us turn our attention to the following operator equation:

$$
A u=M u-f u .
$$

For all $u, \phi \in W_{0}(D)$, we have

$$
A^{\prime} \phi=M \phi-f_{u}(x, u(x)) \phi
$$

If $\inf _{\Omega} f_{u}>\frac{S^{2}}{4 \tau^{2}}$, then zero is not an eigenvalue of $M \phi-f_{u}(x, u(x)) \phi$, so for every $u \in W_{0}(D)$, the operator $A^{\prime}(u)=M-f_{u} I$ is invertible and $A$ is a local homeomorphism from $W_{0}(D)$ onto $L_{2}(D)$, where $I$ denotes the identical operator. Furthermore, an upper bound for || $\left[A^{\prime}(x)\right]^{-1} \| \mid$ is provided by Lemma 3.4 if the coefficient $a(x)$ is identified with $f_{u}(x, u)$, it implies that

$$
\left|\left\|\left[A^{\prime}(x)\right]^{-1} h\right\|\right| \leq\left(\alpha \sup _{\|x\| \leq s}\left|f_{u}(x, u(x))\right|+\beta\right)\|h\|
$$

for positive constant $\alpha, \beta$.

Denote

$$
\lambda(s)=\alpha \sup _{\|x\| \leq s}\left|f_{u}(x, u(x))\right|+\beta,
$$


then

$$
\left|\left\|\left[A^{\prime}(x)\right]^{-1} h\right\|\right|=\left|\left\|\left(M-f_{u}\right)^{-1} h\right\|\right| \leq \lambda(\|u\|)\|h\|, \quad u \in W_{0}(D) .
$$

We may express the first line of (1) in the form

$$
P u=M u-f u+F u=0
$$

for $u, \phi \in W_{0}(D)$, we have

$$
P^{\prime}(u)(\phi)=M \phi-f_{u} \phi+F_{u} \phi .
$$

We can state and prove our main theorem.

Theorem 4.1 In the setting above, for Eqs. (1), (2) and (3) there exists a unique solution if the following conditions hold:

(1) $\inf _{\Omega} f_{u}>\frac{S^{2}}{4 \tau^{2}}$;

(2) for each $\mu \in R$, the maximum solution $y$ of the initial value problem

$$
\left\{\begin{array}{l}
y^{\prime}(t)=\mu \delta(y(t)), \quad t \in[0, a), \\
y(0)=0
\end{array}\right.
$$

is defined on $[0, a]$ and there exists a sequence $t_{n} \rightarrow$ a as $n \rightarrow \infty$ such that $\lim _{n \rightarrow \infty} y\left(t_{n}\right)=y^{\star}$ is finite;

(3) $F$ is continuously differentiable and

$$
\left|\frac{\partial F_{i}}{\partial x_{j}}(w)\right|,\left|\frac{\partial F_{i}}{\partial y_{j}}(w)\right| \leq \frac{c}{n \delta(\|w\|)}, \quad c<1, w \in R^{n} .
$$

Proof Firstly, we prove $\left[M-f_{u}(u)+F_{u}(u)\right]^{-1} \leq \delta(\|u\|)$. For $u, v \in D$, it is obvious that $F$ is continuously Frechet differentiable with

$$
\left[F^{\prime}(u) v\right](t)=-\left(\frac{\partial F_{i}}{\partial x_{j}} u(t)\right) v(t)-\left(\frac{\partial F_{i}}{\partial y_{j}} u(t)\right) v^{\prime}(t)
$$

It follows from the above assumption and (16) that

$$
\left\|F^{\prime}(u) v\right\| \leq n \frac{c}{n \lambda(\|u\|)}\left(\|v\|+\left\|v^{\prime}\right\|\right) \leq \frac{c}{\lambda(\|u\|)}(|\|v\||)
$$

Now

$$
M u-f_{u}+F_{u}=\left(I+F_{u}\left[M-f_{u}\right]^{-1}\right)\left(M-f_{u}\right) .
$$

Let $Q: W_{0}(D) \rightarrow W_{0}(D)$ be defined by

$$
Q=F^{\prime}(u)\left[M-f_{u}\right]^{-1},
$$


by (14) and (16), we have

$$
\|Q h\| \leq \frac{c}{\lambda(\|w\|)} \lambda(\|w\|)\|h\|=c\|h\|, \quad h \in W_{0}(D)
$$

So $I+Q$ is invertible with

$$
\left\|(I+Q)^{-1}\right\| \leq \frac{1}{1-c}
$$

Hence, $M-f_{u}(u)+F_{u}(u): D \rightarrow W_{0}(D)$ is invertible with

$$
\left[M-f_{u}(u)+F_{u}(u)\right]^{-1}=\left[M-f_{u}(u)\right]^{-1}(I+Q)^{-1},
$$

and so

$$
\left[M-f_{u}(u)+F_{u}(u)\right]^{-1} \leq \frac{\lambda(\|u\|)}{1-c}
$$

Denote $\delta(\|u\|)=\frac{\lambda(\|u\|)}{1-c}$, then

$$
\left[M-f_{u}(u)+F_{u}(u)\right]^{-1} \leq \delta(\|u\|)
$$

It implies that $P$ is invertible at every $u \in W_{0}(D)$, hence, $P$ is a local homeomorphism of $W_{0}(D)$.

Secondly, in view of Theorem 2.1, we need only show that $P$ has the property $(C)$ for any continuous function $q:[0,1] \rightarrow L_{2}(D)$. For a given $y \in L_{2}(D)$ and an arbitrary $x_{0} \in W_{0}(D)$, let

$$
q(t)=(1-t) y+t P\left(x_{0}\right)
$$

suppose that there exists a continuous function $r:[0, a) \rightarrow D \subseteq W_{0}(D)$ such that

$$
P(r(t))=q(t), \quad t \in[0, a), \text { for } 0<a \leq 1 .
$$

Now we need to prove that there exists a real sequence $\left\{t_{n}\right\}$ such that $t_{n} \rightarrow a, n \rightarrow \infty$ and

$$
\lim _{n \rightarrow \infty} r\left(t_{n}\right)=r^{\star}
$$

exists and is in $W_{0}(D)$.

It is clear that $r$ is differentiable in this case. We have from (18)

$$
\left\{\begin{array}{l}
r^{\prime}(t)=-P^{\prime}(r(t))^{-1}\left(P\left(x_{0}\right)-P(r(t))\right), \quad t \in[0, a), \\
r(0)=x_{0} .
\end{array}\right.
$$

Denote by $D\|r(t)\|$ the Dini derivative of $\|r(t)\|$ and set $\mu=P(r(t))-P\left(x_{0}\right)$, and we have

$$
D\|r(t)\| \leq\left\|r^{\prime}(t)\right\|=\left\|P^{\prime}(r(t))^{-1}\right\|\left\|P(x)-P\left(x_{0}\right)\right\| \leq \mu \delta(\|r(t)\|) .
$$


By the assumption (2), we know the maximum solution $y(t)$ of $(15)$ is defined on $[0, a)$ and there exists a sequence $t_{n} \rightarrow a$ as $n \rightarrow \infty$ such that

$$
\lim _{n \rightarrow \infty} y\left(t_{n}\right)=y^{\star}
$$

is finite. It follows that $y(t)$ is continuous on $[0, a]$ and there is a constant $K$ such that $|y(t)| \leq K, t \in[0, a]$. By the comparison theorem, Theorem 2.3, we have

$$
\|r(t)\| \leq|y(t)| \leq K, \quad t \in[0, a] .
$$

For $t_{1}, t_{2} \in[0, a)$, we have

$$
\begin{aligned}
\left\|r\left(t_{1}\right)-r\left(t_{2}\right)\right\| & \leq \int_{t_{1}}^{t_{2}}\left\|r^{\prime}(s)\right\| d s \leq \mu \int_{t_{1}}^{t_{2}} \delta(\|r(s)\|) d s \\
& \leq \mu \int_{t_{1}}^{t_{2}} \delta(\|y(s)\|) d s \leq \mu \delta(K)\left|t_{1}-t_{2}\right| .
\end{aligned}
$$

So $\left\{r\left(t_{n}\right)\right\}$ is a Cauchy sequence and consequently for the real sequence $t_{n} \rightarrow a$ as $n \rightarrow \infty$,

$$
\lim _{n \rightarrow \infty} r\left(t_{n}\right)=r^{\star}
$$

exists. This proves that $r^{\star} \in W_{0}(D)$ and $P$ satisfies the condition (C). The theorem is proved.

\section{Related results}

Using a similar technique to the one of the theorem, we can prove the following conclusion as regards the initial-boundary problem for pseudoparabolic equations.

Corollary 5.1 Let the condition (1) and the condition (2) hold and

(3) F be continuously differentiable and

$$
\left|\frac{\partial F_{i}}{\partial x_{j}}(w)\right|,\left|\frac{\partial F_{i}}{\partial y_{j}}(w)\right| \leq \frac{c}{n W}, \quad c<1, w \in R^{n},
$$

where W is constant. Then Eq. (1) has a unique solution.

Corollary 5.2 Let the condition (1) and the condition (2) hold. Assume that $f$ is continuous and has continuous partial derivatives with respect to $u$ through the third order. Then the semilinear equation

$$
\begin{aligned}
& \left(a_{i, j}\left(u+u_{t}\right)_{x_{i}}\right)_{x_{j}}+b_{i} u_{x_{i}}-u_{t}=f(x, t, u), \quad(x, t) \in D, \\
& \left.u\right|_{t=0}=0, \quad x \in \Omega \\
& \left.u\right|_{\partial \Omega}=0, \quad(x, t) \in \partial \Omega \times[0, T]
\end{aligned}
$$

has a unique solution. 
Especially when $a_{i, j}=1, b_{i}=0$, we get the equation in [3]:

$$
\Delta\left(u+u_{t}\right)-u_{t}=f(x, t, u), \quad(x, t) \in D,
$$

and the conclusion of [3] but with a different method.

\section{Acknowledgements}

The authors are grateful to the referees for their comments and references, which improved the paper.

\section{Competing interests}

The authors declare that they have no competing interests.

\section{Authors' contributions}

All authors contributed equally to the manuscript. All authors read and approved the final manuscripts.

\section{Publisher's Note}

Springer Nature remains neutral with regard to jurisdictional claims in published maps and institutional affiliations.

Received: 24 March 2020 Accepted: 7 June 2020 Published online: 22 June 2020

\section{References}

1. Sigillito, V.: On a continuous method of approximating solutions of the heat equation. J. ACM 14(4), 732-741 (1967)

2. Elcrat, A., Sigillito, V.: An explicit a priori estimate for parabolic equations with applications to semilinear equations. J. Math. Anal. 7(3), 746-753 (1976)

3. Elcrat, A., Sigillito, V.: Coercivity for a third order pseudoparabolic operator with applications to semilinear equations. J. Math. Anal. 61(5), 841-849 (1977)

4. Karch, G.: Asymptotic behavior of solution to some pseudoparabolic equation. J. Math. Anal. 20(2), 271-289 (1997)

5. Bouziani, A.: Initial-boundary value problems for a class of pseudoparabolic equations with integral boundary conditions. J. Math. Anal. Appl. 29(2), 371-386 (2004)

6. Bouziani, A., Merazga, N.: Solution to a semilinear pseudoparabolic problem with integral conditions. Electron. J. Differ. Equ. 2006, Article ID 115 (2006)

7. Brown, K.J., Lin, S.S.: Periodically perturbed conservative systems and a global inverse function theorem. Nonlinear Anal. 4(1), 193-201 (1980)

8. Plastock, R.: Homeomorphisms between Banach spaces. Trans. Am. Math. Soc. 200(200), 169-183 (1974)

9. Lakshmikantham, V., Leeda, S.: Solutions to the Cauchy Problem for Differential Equations in Banach Spaces with Fractional Order. Academic Press, San Diego (1969)

\section{Submit your manuscript to a SpringerOpen ${ }^{\circ}$ journal and benefit from:}

- Convenient online submission

- Rigorous peer review

- Open access: articles freely available online

- High visibility within the field

- Retaining the copyright to your article 